Case Report

\title{
A case report of subglottic haemangioma
}

\section{Vignesh Karunakaran $^{1}$, Kutuva Swamirao Rajaganesh ${ }^{2 *}$, Dhinakaran Natarajan ${ }^{2}$}

\author{
Department of Otorhinolaryngology, ${ }^{1}$ Government District Headquarters Hospital, Namakkal, ${ }^{2}$ Madurai Medical \\ College, Madurai, Tamil Nadu, India
}

Received: 14 September 2018

Revised: 18 October 2018

Accepted: 20 October 2018

\section{*Correspondence:}

Dr. Kutuva Swamirao Rajaganesh,

E-mail: rajaganesh.ent@gmail.com

Copyright: (C) the author(s), publisher and licensee Medip Academy. This is an open-access article distributed under the terms of the Creative Commons Attribution Non-Commercial License, which permits unrestricted non-commercial use, distribution, and reproduction in any medium, provided the original work is properly cited.

\begin{abstract}
We report a case of 18 year old female who presented with hoarseness of voice and hemoptysis for 6 months eventually diagnosed as subglottic haemangioma through video laryngoscopy, CT imaging and histopathological examination of excision biopsy. The tumor excision was done by endolaryngeal surgery with tracheostomy under general anesthesia. The diagnosis of subglottic haemangioma, its management and prognosis were discussed.
\end{abstract}

Keywords: Haemangioma, Capillary haemangioma, Subglottic haemangioma

\section{INTRODUCTION}

Hemoptysis is not a common complaint in an otorhinolaryngology clinic, usually benign rarely serious but causes considerable anxiety to the patients. Hemoptysis has a varied etiology, it warrants a thorough investigation based on associated symptoms. ${ }^{1}$ The patient to be discussed here had an associated change of voice which prioritize the ENT examination, in the investigation of hemoptysis.

\section{CASE REPORT}

18 year old female presented to ENT OPD with complaints of change in voice and hemoptysis for 6 months duration. Voice change was insidious, progressive \& hoarse with associated hemoptysis which was episodic, 5-6 events, lesser quantity and no previous history of asthma, tuberculosis, heart disease, and bleeding diathesis. Her physical examination revealed no pallor, normal vesicular breath sounds in all lung fields with no added sounds, no cutaneous lesions. Video laryngoscopy revealed small, reddish lobulated polypoidal pedunculated mass in subglottis on the right side suggestive of haemangioma (Figure 1).
Plain $5 \mathrm{~mm}$ slice CT imaging of neck and chest did not reveal any notable lesion. Laboratory tests revealed normal values of hemoglobin, blood count, coagulation and thyroid profile. Her chest x-ray and sputum AFB results were normal. Planned for the endolaryngeal excision biopsy under general anesthesia through a tracheostomy.

Under local anesthesia tracheostomy was performed, the tracheal cartilage was preserved using superior based tracheal flap and sutured to subcutaneous tissue. General anesthesia was given through tracheostomy, the patient was placed in Boyce position, Jackson laryngoscope passed, fixed with chest suspension. $0^{\circ}$ Hopkins rod was passed, mass visualized in the right side below the vocal cord (Figure 2A), the size and extent were assessed. Local infiltration with $2 \%$ xylocaine with 1: $1,00,000$ adrenaline was given in the base of a tumor. Using a sickle knife, the mass was divided at the base near its attachment \& removed intoto. Tumor site was examined for any remnants, no residual tissue was seen except a minor ooze which was controlled with the application of adrenaline-soaked wick and electrocautery (Figure 2B). Laryngoscope was withdrawn, the patient recovered well 
from general anesthesia. Total procedure duration was 23 minutes.

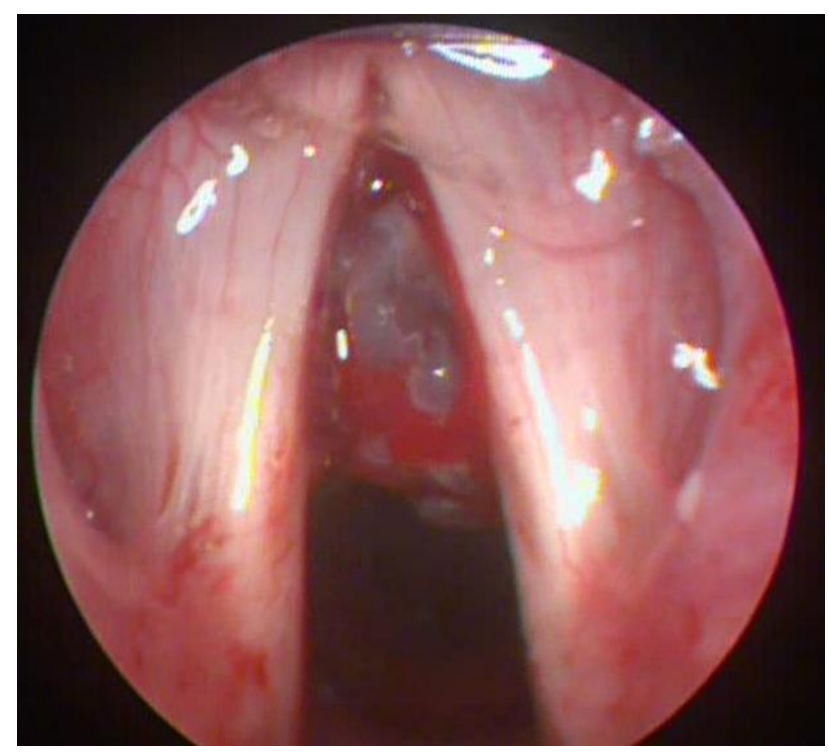

Figure 1: Direct laryngoscopy showing dark red to purplish smooth lobulated mass below the right vocal cord with minimal bleeding over the surface, preventing complete adduction of the vocal cords.

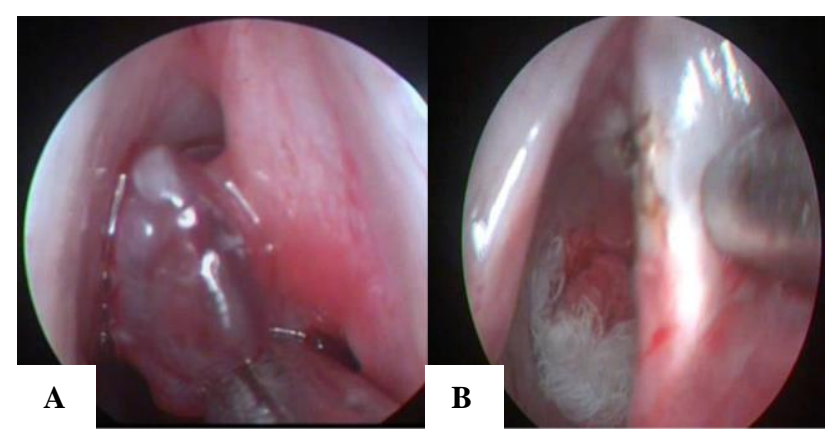

Figure 2: (A) An intraoperative image showing a pedunculated mass below the right vocal cord. (B)

Tumor site after excision of the mass, hemostasis achieved using epinephrine gauze and electrocautery.

Histopathology of the specimen revealed stratified squamous lining epithelium with underlying proliferating capillaries with areas of hemorrhage suggestive of capillary haemangioma.

The immediate postoperative period was uneventful. Video laryngoscopy was done on the $2^{\text {nd }}$ postoperative day, edema was seen at the tumor site and vocal cords movement were normal. $10^{\text {th }}$ post op day repeat video laryngoscopy was done vocal cords structure and movement were normal, subglottis was normal.

Tracheostomy decannulation was done in operation theatre, superior based flap repositioned; the stoma wound was closed with subcuticular sutures. Postoperative voice evaluation was normal. With a tumor being removed and voice restored, the patient was managed successfully.

\section{DISCUSSION}

Haemangioma is a common benign tumor of the head and neck in children which usually appears a few weeks after birth, grows more rapidly during infancy, and undergoes spontaneous slow involution later in childhood. ${ }^{2}$ There are several histologic and clinical variants: capillary haemangioma, cavernous haemangioma, and pyogenic granuloma. Capillary haemangioma is the most common variant of haemangioma which occurs in the skin, subcutaneous tissues, and mucous membranes of the oral cavities, lips, as well as in internal viscera.

In an old literature series, Sweetser classified haemangioma of the airways into an infantile and an adult variety. ${ }^{3}$ The infantile type is usually in the subglottic airways, rarely involving subcricoid area and usually develop dyspnoea and stridor by three months. The adult types of hemangiomas are usually supraglottic, commonly involving the vocal cords. Hoarseness with little or no dyspnoea is the most common manifestation, and hemoptysis occurs frequently.

Hemoptysis in adolescents is more frequently caused by infection, congenital heart disease, neoplasms, and bronchiectasis. ${ }^{4}$

The tumor size being smaller is most likely to be missed in the CT imaging, proving video laryngoscopy an essential tool in the diagnosis. ${ }^{5}$ But CT imaging gave useful information of rest of airway, neck, and parenchyma of the lung thereby aid in planning the management.

Based on the site and appearance, the most probable diagnosis would be haemangioma though papilloma, leiomyoma, adenoma, mucoepidermoid carcinoma, and any benign lesions could be considered. ${ }^{6}$

Analysing the various anesthetic methods for laryngeal surgeries such as supraglottic jet venturi needle, microlaryngeal tube, and subglottic jet ventilation. We preferred tracheostomy as it holds the advantages of improved surgical access, management of hemorrhage, airway management in case postoperative vocal fold edema due to use of cautery and postoperative voice rest.

\section{CONCLUSION}

There is no established management protocol for adult capillary haemangioma. Role of medical management in adult cases are not studied due rarity of presentation and it is easily amenable to surgical management. Various modalities of treatment are available such as laryngoscopy excision, argon plasma coagulation, embolization, laser, etc., which are individualized based on size, location, and comorbidities. Laryngoscopy/ 
bronchoscopy forceps excision gave better results with no recurrence even after three years follow up in our case.

Funding: No funding sources

Conflict of interest: None declared

Ethical approval: Not required

\section{REFERENCES}

1. Bannister M. Paediatric haemoptysis and the otorhinolaryngologist: Systematic review. Int $\mathrm{J}$ Pediatr Otorhinolaryngol. 2017;92:99-102.

2. Rose AS, Mathur PN. Endobronchial capillary hemangioma: case report and review of the literature. Respir Int Rev Thorac Dis. 2008;76(2):221-4.

3. Sweetser TH. Hemangioma of the larynx. The Laryngoscope. 1921;31(10):797-806.

4. Sim J, Kim H, Lee H, Ahn K, Lee SI. Etiology of hemoptysis in children: a single institutional series of 40 cases. Allergy Asthma Immunol Res. 2009;1(1):41-4.

5. Amy FT, Enrique D-G. Lobular Capillary Hemangioma in the Posterior Trachea: A Rare Cause of Hemoptysis. Case Reports in Pulmonology. 2012. Available at: https://www. hindawi.com/journals/cripu/2012/592524/. Accessed on 23 July 2017.

6. Varela P, Pio L, Brandigi E, Paraboschi I, KhenDunlop N, Hervieux E, et al. Tracheal and bronchial tumors. J Thorac Dis. 2016;8(12):3781-6.

Cite this article as: Karunakaran V, Rajaganesh KS, Natarajan D. A case report of subglottic haemangioma. Int J Otorhinolaryngol Head Neck Surg 2019;5:209-11. 